\title{
REGIONAL AND UNIVERSITY ROLE IN BIOTERRORISM PREPAREDNESS
}

\author{
Steven Hinrichs \\ Director, Nebraska Public Health Lab \\ University of Nebraska Medical Center
}

Thank you for inviting me to make this presentation. I am currently a Professor in the Department of Pathology and Microbiology at the University of Nebraska Medical Center (UNMC). In addition, I have a second position as Director of the Nebraska Public Health Laboratory (NPHL). Our relationship with the Nebraska Health and Human Services systems enables diagnostic procedures to be performed under a contractual relationship with the State of Nebraska. This system is unique in that NPHL is fully integrated into the University and its clinical partner.

I would like to modify my presentation in response to previous talks and address the regional and university role in biocrime or biosecurity preparedness. I agree with our earlier speaker that we must transition from shock and fear associated with anthrax and the bombing, and develop an appropriate effort to prevent these occurrences in the future.

While some aspects of the NPHL at UNMC are unique, we share many features in common with academic medical centers in the Midwest. For example, we are a bone marrow and solid organ transplantation facility. We operate a radiation health center that provides emergency services to the nuclear power plant facilities in our region. We also maintain and operate high-risk isolation facilities for patients who have been exposed to highly infectious agents such as those on the select agent list. These are functions that exist at other university medical centers. Because we have a common base of experience and significant capabilities, our regional medical centers and land grant universities could come together and develop an interactive program that benefits our country and our region. The challenges faced by our nation are the same challenges that universities in our region are fully prepared to undertake.

Many of the pathogens we face are capable of causing disease in both humans and animals. In addition, a threat to water quality is a threat to both the medical community and the agricultural community and, of course, a threat to crop production and food production is a threat to all living things. An adequate response to these types of threats requires a high degree of interaction between experts at all levels whether they deal with water, food crop production, veterinary sciences, basic research or medical treatment of humans. The universities represented here are well positioned to interact and develop a unified approach to these challenges. 
Our challenges can be summarized under four topic areas: genetics, immunology, novel therapeutics, and diagnostic modalities. These topics are typical for developmental or research programs at our institutions. In addition, we share common opportunities in new cutting-edge technologies, such as information exchange and data analysis, and we all have the responsibility to educate students and the public. My associates have recently developed and made use of microarray technology for the rapid application to infectious diseases. One of the most difficult challenges is that of rapid triaging distinguishing the possible cause of disease and determining whether it is bacterial, viral or fungal in origin. By using microarray technology, the distinction can be rapidly determined. One of the new areas we are investigating is the interaction and collaboration between researchers involved in diagnostic test development and those involved in large-scale test automation. There are a number of opportunities for applying molecular technology to organism identification using automated procedures. One specific application might be the use of multiplex PCR for the detection of enterohemorrhagic $E$. coli associated genes in various patient materials as well as foods. The automated technology is necessary to reduce the cost so that it approximates standard culture technologies. Although we may never be able to completely approximate culture technique costs, molecular approaches offer another benefit - rapid availability of results.

A cooperative program between our regional medical schools, veterinary schools, and agricultural colleges would be a highly effective approach for utilizing animal models for the study of vaccine efficacy as well as identification of virulence factors within organisms on the select agent list. Through the development of a regional consortium, it might be possible to rapidly investigate and determine the pathogenesis of various diseases and develop appropriate vaccines. Beyond this, a cooperative regional approach is needed to take advantage of new developments in information technology. The Centers for Disease Control and Prevention (CDC) has done the nation a great service by developing standards for exchange of electronic information. All of us have a credit card that can be used in any number of stores or restaurants, but I challenge you to pull out of your wallet a medical information card that can instantly download your relevant medical records. The reason a card does not exist is largely due to the lack of standards allowing it to be used and produced. Through the development of standards and the creation of a National Electronic Disease Surveillance System (NEDSS), the CDC is working to address this problem. We must be prepared to take advantage of the opportunity it presents.

While much has been said about the disease monitoring capabilities possible through syndromic surveillance programs, I also think they represent a significant problem that will result in less functionality than expected. Syndromic surveillance commonly refers to the detection of a pattern of diseases from patients appearing in emergency rooms or doctors' offices. Since much of this 
information is required to be anonymous, it will be difficult to determine what level of investigation should be prompted by an upturn in certain disease types, and what those active interventions should or could be. However, our universities already have the expertise and capability to develop algorithms and mathematical models for determining the efficacy and usability of this information. Therefore, we must leverage our resources and make them available for addressing the problem of disease surveillance. Information technology is not the only resource our universities have available; we also have highly capable statewide information services at our county extension offices as well as area health education centers for the training of medical students and residents. Education is our number one job. We should educate the public by using the skills and capabilities we have developed to address problems on the farm or ranch, and in medical practice.

We all recognize the many challenges raised by the threat of bioterrorism. The Heartland of America has its own regional concerns and challenges, and politics is a reality here as well. On the other hand, many of the traditional obstacles and political problems encountered in large metropolitan areas of the country may not be obstacles to a Midwestern research consortium on biosecurity. I'll leave this issue, however, to those experts in the university chancellors' offices and to our distinguished visitors here. I do believe a cooperative strategy would be highly competitive with other programs put forward by the large private research universities on the east or west coast.

In closing, I would like to thank Mr. Keith Yehle for his comments and suggestions regarding delivery of anthrax-contaminated mail to the Senate Hart office building. Thank you, Dr. Mabel Rice, for the opportunity to attend this meeting and give this presentation. 